\title{
Certifying diabetes-related cause-of-death: a comparison of inappropriate certification statements in Sweden, Taiwan and the USA
}

\author{
T. H. Lu • P. Y. Hsu • C. Bjorkenstam • R. N. Anderson
}

Received: 28 April 2006 / Accepted: 1 September 2006 / Published online: 10 October 2006

(C) Springer-Verlag 2006

\begin{abstract}
Aims/hypothesis The aim of this study was to assess differences in the certification practices of physicians in Sweden, Taiwan and the USA with regard to diabetesrelated cause-of-death (COD) statements.

Methods Multiple-cause-of-death data from Sweden (2000), Taiwan (2001) and the USA (2001) were used for this study. All deaths with mention of diabetes anywhere on the death certificate were extracted for analysis. Two types of inappropriate COD statements were: (1) reporting two or more diagnoses per line; and (2) entering an incorrect causal sequence among reported diagnoses.

Results Of those deaths in which diabetes was reported in Part I of the death certificate, American physicians (19\%) were less likely to report two or more diagnoses per line than physicians in Sweden (46\%) and Taiwan (56\%). On the other hand, Swedish physicians (5\%) were less likely to
\end{abstract}

\section{T. H. Lu}

Institute of Public Health, College of Medicine,

National Cheng Kung University,

Tainan, Taiwan

P. Y. Hsu

Office of Statistics, Department of Health,

Taipei, Taiwan

C. Bjorkenstam

Centre for Epidemiology, National Board of Health and Welfare,

Stockholm, Sweden

R. N. Anderson $(\bowtie)$

Mortality Statistics Branch, Division of Vital Statistics,

National Center for Health Statistics,

Centers for Disease Control and Prevention,

3311 Toledo Road, Room 7318,

Hyattsville, MD 20782, USA

e-mail: rca7@cdc.gov report incorrect causal sequences than were their counterparts in Taiwan (21\%) and the USA (28\%).

Conclusions/interpretation These findings reveal substantial differences in diabetes-related COD statements among physicians in Sweden, Taiwan and the USA, implying that caution should be used when interpreting differences in mortality statistics between these countries.

Keywords Cause-of-death · Death certificates .

Diabetes mellitus · Mortality

\author{
Abbreviations \\ ACME Automated Classification of Medical Entities \\ COD cause-of-death \\ ICE International Collaborative Effort \\ UCOD underlying-cause-of-death
}

\section{Introduction}

International comparisons of mortality data provide an important resource for formulating hypotheses for epidemiological studies. To achieve valid international comparisons, the WHO established a standard cause-of-death (COD) certification format [1]. The instructions indicate that the diagnosis in line (a) is due to the diagnosis in line (b), which is due to the diagnosis in line (c), etc., with the last used line in Part I indicating the underlying-cause-ofdeath (UCOD). The UCOD is defined as the disease or injury which initiated the train of morbid events leading directly to death'. The UCOD is used to tabulate official mortality statistics [1]. While the format for COD certification is standardised, physicians often fail to follow instructions and record the COD statement improperly [2, 3]. 
The problems are particularly noteworthy for diabetesrelated deaths, because it is not always obvious whether diabetes should appear in the causal sequence or whether it should be reported in Part II as a contributing condition [4, 5].

The present study is part of the International Collaborative Effort (ICE) on Automating Mortality Statistics initiated by the US National Center for Health Statistics [6]. One important goal of the ICE is to promote better comparability of mortality statistics between countries. Evaluating the comparability of diabetes-related mortality across countries is one important topic in the ICE. Previous studies associated with the ICE have also revealed that physicians from different countries tend to have different habits with regard to the reporting of diabetes on the death certificate, e.g. whether diabetes is reported in Part I or Part II [7], and whether associated cardiovascular diseases are reported along with diabetes [8]. These differences can have a significant effect on international comparisons of diabetes mortality. The aim of this study was to assess differences in diabetes-related COD statements that tend to substantially affect the selection of the UCOD.

\section{Subjects and methods}

Electronic multiple-cause-of-death data for Sweden (2000), Taiwan (2001) and the USA (2001) were used for analysis. All three countries use the same automated coding system, i.e. Automated Classification of Medical Entities (ACME), to assign the UCOD, by which the inter-coder variability in the selection of the UCOD could be eliminated. ACME records each entered diagnosis and its relative location on the death certificate. With this kind of detailed information in electronic form we were able to analyse the large amount of physicians' COD statements with the help of computer algorithms [9].

The following two inappropriate diabetes-related COD statements were examined because they are likely to result in variation in the selection of the UCOD.

Reporting two or more diagnoses per line in Part I of the death certificate We used the information of the exact location of each diagnosis on the death certificate to determine if the certifying physician reported two or more diagnoses per line in Part I, e.g. line (b) in Examples 1 and 2 (Table 1). The more diagnoses reported per line, the more the selection rules come into play when selecting the UCOD and the more likely that the UCOD selected according to the rules did not reflect the real intention of the physician.

Reporting incorrect causal sequences among reported diagnoses in Part I of the death certificate We first used an algorithm to single out death certificates in which the selection of the UCOD was not based on the General
Table 1 Examples of inappropriate cause-of-death statements in Part I of the death certificate

\begin{tabular}{lll}
\hline Example & Line & Diagnosis \\
\hline 1 & a & Congestive heart failure \\
Myocardial infarction, \\
cerebrovascular infarction
\end{tabular}

Principle. The algorithm compared the first diagnosis in the last used line in Part I (i.e. hypertension in Example 1 and Example 2, diabetes in Example 3 [Table 1]) with the UCOD ultimately selected by ACME (i.e. hypertension in Example 1, diabetes in Example 2 and lung cancer in Example 3). For those cases with differences (e.g. Example 2 and Example 3), the first two authors (T. H. Lu and P. Y. $\mathrm{Hsu}$ ) then checked the ACME process messages to confirm if the causal sequences among reported diagnoses were highly improbable. The key ACME process message to confirm the incorrect causal sequence in Example 2 and 3 can be illustrated as follows: Example 2: Is E419 (diabetes) due to I10 (hypertension)? NO; Example 3: Is C62 (lung cancer) due to E419 (diabetes)? NO.

The confirmation was done by checking with the help of a computer whether there was 'NO' in the ACME process message for each case.

The incorrect causal sequences were further classified into two types: (1) other diagnoses incorrectly reported as the cause(s) of diabetes - e.g. hypertension incorrectly reported as the cause of diabetes in Example 2; and (2) diabetes incorrectly reported as the cause of other diagnoses - e.g. diabetes incorrectly reported as the cause of lung cancer in Example 3.

\section{Results}

During the periods included in this study, 93,461 death certificates were issued in Sweden in 2000, 126,667 in Taiwan in 2001 and 2,419,960 in the USA in 2001. Diabetes was mentioned in a similar percentage of death certificates in Sweden $(9 \%$; 8,255 of 93,461), Taiwan (10\%; $12,827$ of 126,667$)$ and the USA $(9 \% ; 218,347$ of $2,419,960)$. However, in these cases, Taiwanese physicians were much more likely to report diabetes in Part I of the death certificates $(70 \% ; 8,934$ of 12,827$)$ than their 
counterparts in Sweden $(21 \% ; 1,728$ of 8,255$)$ and the USA $(36 \% ; 79,488$ of 218,347$)$.

As shown in Table 2, of those deaths for which diabetes was reported in Part I of the death certificates, American physicians $(19 \% ; 15,278$ of 79,488$)$ were much less likely to report two or more diagnoses per line than physicians in Sweden $(46 \% ; 794$ of 1,728$)$ and Taiwan $(56 \% ; 4,958$ of $8,934)$. Swedish physicians $(5 \% ; 79$ of 1,728$)$ were less likely to report incorrect causal sequences than were their counterparts in Taiwan $(21 \% ; 1,893$ of 8,934$)$ and the USA $(28 \% ; 21,835$ of 79,488$)$.

About three-quarters of the incorrect causal sequences involved incorrectly reporting other conditions (mainly cardiovascular diseases) as the cause of diabetes. Hypertension and acute myocardial infarction were the two conditions most often reported as the cause of diabetes. Diabetes was more likely to be incorrectly reported as the cause of infectious diseases, such as HIV and tuberculosis, cancers and chronic obstructive pulmonary diseases.

\section{Discussion}

Our findings reveal important differences in diabetesrelated COD statements for Sweden, Taiwan and the USA. American physicians were less likely than those in Sweden and Taiwan to report two or more diagnoses per line. Swedish physicians, on the other hand, were less likely to report incorrect causal sequences than their counterparts in Taiwan and the USA.

Why do physicians often report two or more diagnoses per line? A likely explanation is that multiple diseases and complications often co-exist with diabetes at the time of death, which makes the determination of a single causal sequence difficult. A previous study revealed that if there were two competing CODs, e.g. diabetes and acute myocardial infarction, or a chronic disease with acute complications, e.g. diabetes with urinary tract infection resulting in sepsis, the variations in COD statements among participating physicians increased [10]. Therefore, multiple diseases in one line may reflect the certifier's uncertainty about the UCOD determination.

What is the impact of certifying two or more diagnoses per line? This certification practice would complicate the selection of the UCOD and, as a result, the selected UCOD might not reflect the real intention of the certifying physician. It may be that the physician's intended UCOD in Example 1 was diabetes; however, he/she reported diabetes as the second diagnosis on the last used line. As a result, the UCOD selected by ACME would not be diabetes.

A possible explanation for the relatively high percentage of incorrect causal sequences among reported diagnoses is that physicians tend to overlook the instructions. Another possibility is that some physicians copy the layout of the discharge diagnoses from the medical record directly onto the death certificate. The layout of discharge diagnoses is based on the reason for admission and the severity of diseases, which is quite different from the logic on which the UCOD is based.

One limitation of this study was that the quality of data transfer from death certificates to the ACME system might be different in the three countries studied. Each country routinely sampled some death certificates to assess typographical errors in transferring reported information on death certificates, but no exact figures of reliability were available.

In conclusion, we found substantial international differences in certification practices regarding diabetes-related COD statements among physicians in Sweden, Taiwan and the USA. Given that such inappropriate COD statements can have a substantial impact on UCOD selection, differences in diabetes mortality statistics between the three countries should be interpreted cautiously. This study also highlights the use of ACME to help to identify questionable death certificates for query back to the certifying physicians. Clarification of the intention of the certifier in cases where the COD statement is incorrectly or unclearly reported has the potential to significantly improve the overall quality of COD statistics.

Table 2 Frequency of inappropriate diabetes-related cause-of-death statements among those deaths in which diabetes was reported in Part I of the death certificate: Sweden, 2000; Taiwan, 2001; and USA, 2001

\begin{tabular}{|c|c|c|c|c|c|c|}
\hline \multirow[t]{2}{*}{ Cause-of-death statements } & \multicolumn{2}{|c|}{ Sweden } & \multicolumn{2}{|c|}{ Taiwan } & \multicolumn{2}{|l|}{ USA } \\
\hline & $n$ & $\%$ & $n$ & $\%$ & $n$ & $\%$ \\
\hline Diabetes reported in Part I of the death certificate & 1,728 & 100 & 8,934 & 100 & 79,488 & 100 \\
\hline Reporting two or more diagnoses per line & 794 & 45.9 & 4,958 & 55.5 & 15,278 & 19.2 \\
\hline Incorrect causal sequence among reported diagnoses & 79 & 4.6 & 1,893 & 21.2 & 21,835 & 27.5 \\
\hline Diabetes incorrectly entered as cause of other conditions & 17 & 1.0 & 585 & 6.5 & 4,792 & 6.0 \\
\hline Other condition incorrectly entered as cause of diabetes & 62 & 3.6 & 1,308 & 14.6 & 17,043 & 21.4 \\
\hline
\end{tabular}


Acknowledgement This study was partially funded by the Taiwan National Science Council (grant NSC93-2320-B-006-051).

Duality of interest No duality of interest exists for any of the authors.

\section{References}

1. World Health Organization (1992) International statistical classification of diseases and related health problems, 10th revision. World Health Organization, Geneva

2. Maudsley G, Williams EMI (1996) 'Inaccuracy' in death certification - where are we now? J Public Health Med 18:59-66

3. Lu TH, Shou WY, Shih TP, Lee MC, Chou MC, Lin CK (2001) Factors associated with errors in death certificate completion: a national study in Taiwan. J Clin Epidemiol 54:232-238

4. Will JC, Vinicor F, Stevenson J (2001) Recording of diabetes on death certificates: has it improved? J Clin Epidemiol 54:239-244
5. Muhlhauser I, Sawicki PT, Blank M, Overmann H, Richter B, Berger M (2002) Reliability of cause of death in persons with type I diabetes. Diabetologia 45:1490-1497

6. National Center for Health Statistics (2006) Initiative and other activities. International Collaborative Effort on Automating Mortality. Available from http://www.cdc.gov/nchs/about/otheract/ice/ automort/automort.htm, accessed 5 August 2006

7. $\mathrm{Lu} \mathrm{TH}$, Walker S, Johansson LA, Huang CN (2005) An international comparison study indicated physicians' habits in reporting diabetes in Part I of death certificate affected reported national diabetes mortality. J Clin Epidemiol 58:1150-1157

8. Lu TH, Hsu PY, Anderson RN, Huang CN (2005) Response to Tseng 'Mortality and causes of death in a national sample of diabetic patients in Taiwan'. Diabetes Care 28:1266-1267

9. Lu TH (2003) Using ACME (Automatic Classification of Medical Entry) software to monitor and improve the quality of cause of death statistics. J Epidemiol Community Health 57:470-471

10. Lu TH, Shih TP, Lee MC, Chou MC, Lin CK (2001) Diversity in death certification: a vignette approach. J Clin Epidemiol 54:1086-1093 